\title{
GRANULATION SIGNATURES IN THE SPECTRUM OF THE VERY METAL-POOR RED GIANT HD 122563
}

\author{
I. Ramírez ${ }^{1,2}$, R. Collet ${ }^{2}$, D. L. Lambert ${ }^{3}$, C. Allende Prieto ${ }^{4,5}$, And M. Asplund ${ }^{2}$ \\ ${ }^{1}$ The Observatories of the Carnegie Institution for Science, 813 Santa Barbara Street, Pasadena, CA 91101, USA \\ ${ }^{2}$ Max Planck Institut für Astrophysik, Postfach 1317, 85741 Garching, Germany \\ ${ }^{3}$ McDonald Observatory and Department of Astronomy, University of Texas, Austin, TX 78712-0259, USA \\ ${ }^{4}$ Instituto de Astrofísica de Canarias, 38205, La Laguna, Tenerife, Spain \\ 5 Departamento de Astrofísica, Universidad de La Laguna, 38206, La Laguna, Tenerife, Spain \\ Received 2010 October 26; accepted 2010 November 16; published 2010 December 6
}

\begin{abstract}
A very high resolution $(R=\lambda / \Delta \lambda=200,000)$, high signal-to-noise ratio $(\mathrm{S} / \mathrm{N} \simeq 340)$ blue-green spectrum of the very metal-poor $([\mathrm{Fe} / \mathrm{H}] \simeq-2.6)$ red giant star HD 122563 has been obtained by us at McDonald Observatory. We measure the asymmetries and core wavelengths of a set of unblended $\mathrm{Fe}$ I lines covering a wide range of line strength. Line bisectors exhibit the characteristic C-shape signature of surface convection (granulation) and they span from about $100 \mathrm{~m} \mathrm{~s}^{-1}$ in the strongest Fe I features to $800 \mathrm{~m} \mathrm{~s}^{-1}$ in the weakest ones. Core wavelength shifts range from about -100 to $-900 \mathrm{~m} \mathrm{~s}^{-1}$, depending on line strength. In general, larger blueshifts are observed in weaker lines, but there is increasing scatter with increasing residual flux. Assuming local thermodynamic equilibrium (LTE), we synthesize the same set of spectral lines using a state-of-the-art three-dimensional (3D) hydrodynamic simulation for a stellar atmosphere of fundamental parameters similar to those of HD 122563. We find good agreement between model predictions and observations. This allows us to infer an absolute zero point for the line shifts and radial velocity. Moreover, it indicates that the structure and dynamics of the simulation are realistic, thus providing support to previous claims of large 3D-LTE corrections to elemental abundances and fundamental parameters of very metal-poor red giant stars obtained with standard 1D-LTE spectroscopic analyses, as suggested by the hydrodynamic model used here.
\end{abstract}

Key words: stars: atmospheres - stars: individual (HD 122563) - stars: Population II

Online-only material: color figures

\section{INTRODUCTION}

The red giant star HD 122563 is the brightest very metal-poor $([\mathrm{Fe} / \mathrm{H}] \lesssim-2.5)$ object known $(V=6.2 \mathrm{mag})$. Its extreme metal deficiency was first identified by Wallerstein et al. (1963) and since then a large number of studies concerning its detailed chemical composition have been published (e.g., Sneden 1973; Lambert et al. 1974; Ryan et al. 1996; Cayrel et al. 2004). Twenty entries more recent than 1990 are listed for HD 122563 in the PASTEL database of stellar parameters (Soubiran et al. $2010)$, which give $T_{\text {eff }}=4602 \pm 72 \mathrm{~K}, \log g=1.25 \pm 0.22$, and $[\mathrm{Fe} / \mathrm{H}]=-2.64 \pm 0.12$ (mean and standard deviation).

Most elemental abundance studies use one-dimensional (1D) model atmospheres in hydrostatic equilibrium. The atmospheres of cool stars, however, are far from static. Velocity and intensity fluctuations due to surface convection are directly observed in the Sun (e.g., Nesis et al. 1992; Rutten et al. 2004) and have been detected indirectly using high-resolution spectra of a number of other stars (e.g., Allende Prieto et al. 2002; Gray 2005; Dravins 2008; Ramírez et al. 2008). In the solar photosphere, bright granules containing hot ascending gas are observed, separated by intergranular lanes of cool descending gas. The correlation between temperature and velocity fields, as well as the granule/intergranule contrast in velocity and intensity, shapes the spectral features so that the line cores of disk-integrated profiles are shifted with respect to their rest wavelengths and the line profiles are asymmetric (e.g., Dravins et al. 1981). The latter are granulation signatures that can be observed both in the solar spectrum and in the spectra of distant stars.

Hydrodynamic simulations provide the most realistic approach to the modeling of surface inhomogeneities (e.g.,
Nordlund 1982; Steffen \& Freytag 1991; Ludwig et al. 2009; Nordlund et al. 2009), although non-hydrodynamic modeling is also possible (e.g., Dravins 1990). Three-dimensional (3D) model atmospheres for the Sun, Procyon, and K-dwarfs have been tested against high-quality spectroscopic data (Asplund et al. 2000; Allende Prieto et al. 2002; Ramírez et al. 2009), showing in general good agreement. These tests are crucial because they validate the models and allow us to use them with confidence to estimate the impact of "3D effects" on stellar abundance and parameter determinations (Asplund 2005).

The most extreme abundance corrections proposed in connection with granulation effects occur at low metallicities. This is mainly because the difference between the $1 \mathrm{D}$ and $3 \mathrm{D}$ predictions for the mean temperature of the outer layers of metal-poor stars is very large, on the order of $1000 \mathrm{~K}$ (e.g., Collet et al. 2006, 2007; González Hernández et al. 2010). This large temperature difference is very important for the formation of features that are temperature sensitive. For example, Collet et al. (2009) report $3 \mathrm{D}$ corrections of $\sim-1$ dex (i.e., one order of magnitude) for the abundance of nitrogen and oxygen as determined from molecular features, similar to the corrections required for the iron abundance determined from low excitation potential $\mathrm{Fe}$ I lines. They also estimate a correction of about +0.4 dex for the iron abundance determined from Fe II lines.

We can only rely on the corrections suggested by 3D models if the hydrodynamic simulations are realistic. Tests of the hydrodynamic simulations include measurements of the profiles of stellar absorption lines both with respect to shape and absolute wavelength. In this Letter, we present new spectroscopic observations for the very metal-poor red giant star HD 122563 and compare them to state-of-the-art 3D model predictions. 


\section{SPECTROSCOPIC OBSERVATIONS, LINE DATA, AND MEASUREMENT OF LINE ASYMMETRIES}

We observed HD 122563 on 2010 June 21-25, with the Robert G. Tull coudé spectrograph on the $2.7 \mathrm{~m}$ Harlan J. Smith Telescope at McDonald Observatory. Using a narrow slit ( $0.34 \mathrm{arcsec}$ ), the spectral resolution is $R=200,000$ (Tull et al. 1995). The wavelength coverage of a single exposure consists of 16 non-overlapping pieces of $\simeq 20 \AA$ each. To increase wavelength coverage we rotated the spectrograph grating to three different positions (setups). In this way, we were able to cover about $70 \%$ of the $4425-5575 \AA$ spectral window. To increase the signal-to-noise ratio $(\mathrm{S} / \mathrm{N})$, multiple 20 minute exposures of each setup (from 8 to 16, depending on the setup) were acquired. The total exposure time for all setups was about $10.5 \mathrm{hr}$. The $\mathrm{S} / \mathrm{N}$ of the co-added spectrum increases from about 180 at $4425 \AA$ to 440 at $5575 \AA$, with a median $\mathrm{S} / \mathrm{N}=340$. The spectroscopic data were reduced and co-added as in Ramírez et al. (2008). The rms of the wavelength calibration for each exposure is about $5 \times 10^{-4} \AA\left(\simeq 30 \mathrm{~m} \mathrm{~s}^{-1}\right)$. Continuum normalization was done by fitting polynomials to the upper envelope of the data, first in the direction of dispersion and then perpendicular to it, as in Barklem et al. (2002). The barycentric correction was determined with a precision better than $1 \mathrm{~m} \mathrm{~s}^{-1}$ using a program by S. Els (2008, private communication).

A radial velocity correction was applied to each setup by comparing the observed core wavelength shift versus line strength relation with the theoretical one (cf. Section 4). This procedure is similar to that described in Gray (2009) but using a theoretical prediction instead of a solar reference. Basically, the zero point of the observed core wavelength shifts is modified until the average difference with the model, for all lines in a given setup, is exactly zero. The absolute radial velocity we derive by applying this method to the three setups is $V_{r}=-25.39 \pm$ $0.09 \mathrm{~km} \mathrm{~s}^{-1}$ (the error bar corresponds to the $1 \sigma$ standard deviation of the three velocities derived). ${ }^{6}$ The uncertainty in this velocity is most likely due to instrumental and temperature/ pressure instabilities (the R. G. Tull spectrograph is not in a vacuum).

We identified 58 unblended Fe I lines in our HD 122563 spectrum. For these features we adopted the laboratory wavelengths measured by Nave et al. (1994), which have a precision of about $20 \mathrm{~m} \mathrm{~s}^{-1}$, as estimated from the uncertainty in the wavenumber of the relevant energy levels. The line synthesis presented in Section 3 was done using transition probabilities measured in the laboratory by a number of groups and homogenized following the recommendations by Lambert et al. (1996). The van der Waals damping constants used are those by Barklem et al. (2000), except for five lines in which we used the values obtained from the classical Unsöld formula.

Line bisectors were measured in our HD 122563 spectrum (and also in an identical fashion in the theoretical line profiles, Section 3) by first resampling the spectral lines, using cubic spline interpolations, to iso-flux points on both wings of the lines. The midpoints of horizontal segments across the spectral line wings, which define the line bisector, were then computed.

\footnotetext{
6 This absolute radial velocity is not corrected for the star's gravitational redshift, which is, however, expected to be relatively small (e.g., Dravins et al. 1999). Metal-poor red giants have typical masses of $\simeq 0.75 M_{\odot}$ while we estimate a radius of $\simeq 23 R_{\odot}$ for HD 122563 based on its observed Hipparcos parallax (van Leeuwen 2007) and the angular diameter obtained with the infrared flux method (Ramírez \& Meléndez 2005). These values lead to a gravitational redshift of $633 \mathrm{~m} \mathrm{~s}^{-1} \times\left[\left(M / M_{\odot}\right) /\left(R / R_{\odot}\right)\right] \simeq 0.021 \mathrm{~km} \mathrm{~s}^{-1}$ which is smaller than our uncertainty in $V_{r}$.
}

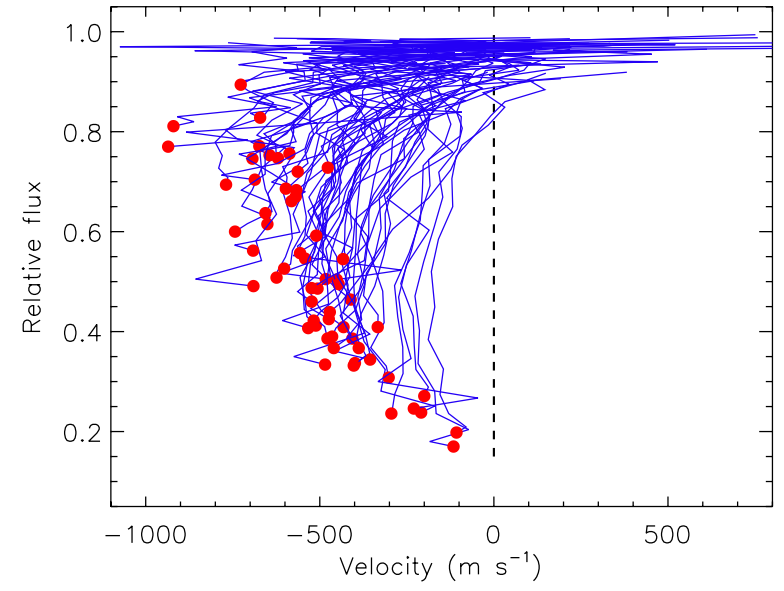

Figure 1. Bisectors of $58 \mathrm{Fe}$ I lines in our spectrum of HD 122563. Filled circles show the location of the line cores. The dashed line is at zero velocity.

(A color version of this figure is available in the online journal.)

The wavelengths of observed spectral line cores were determined using the 7 data points closest to the flux minimum, which corresponds to a window of about $3.8 \mathrm{~km} \mathrm{~s}^{-1}$, and fitting a fourth-order polynomial. Core wavelength shifts (hereafter given as a velocity, i.e., $\Delta v_{c}=c \Delta \lambda / \lambda$, where $c$ is the speed of light and $\Delta \lambda=\lambda_{\text {obs }}-\lambda_{\text {lab }}$ ) were then derived using the adopted laboratory wavelengths. Observational errors for the line bisectors were computed following Gray (1983).

The observed line bisectors are shown in Figure 1, where we emphasize the location of the line cores. The cores of the strongest lines are blueshifted by about $-100 \mathrm{~m} \mathrm{~s}^{-1}$ while those of weaker lines show more blueshift, up to $-900 \mathrm{~m} \mathrm{~s}^{-1}$ (note that the absolute velocity scale was determined using 3D model predictions, as explained in Section 4). The overall shape of the bisectors resembles the letter $\mathrm{C}$, although it varies with line strength. Strong (weak) lines resemble more the lower (upper) part of the letter $C$. This is not very different from the case of cool metal-rich stars, implying that low metallicity does not affect the basic behavior of bisector shapes (see also Gray et al. 2008). The strongest lines in the spectrum of HD 122563 (residual flux of about 0.2) are more symmetric and their bisectors have a span of about $100 \mathrm{~m} \mathrm{~s}^{-1}$. The weaker lines (residual flux of about 0.8 ), on the other hand, have a bisector span of about $800 \mathrm{~m} \mathrm{~s}^{-1}$. In a hydrostatic atmosphere, the lines would be symmetric and not shifted. This hypothetical case is illustrated with the dashed line in Figure 1.

\section{HYDRODYNAMIC SIMULATION}

R. Collet et al. (2011, in preparation; see also Collet et al. 2009) used the STAGGER code by Nordlund \& Galsgaard (1995) to compute a 3D radiative-hydrodynamic model with stellar parameters $T_{\text {eff }}=4600 \mathrm{~K}, \log g=1.6(\mathrm{cgs})$, and scaled solar chemical composition with $[\mathrm{Fe} / \mathrm{H}]=-3$. The stellar parameters were taken from Barbuy et al. (2003) with a slight revision of the surface gravity based on the parallax determination of HD122563 from the Hipparcos data reduction by van Leeuwen (2007).

The 3D model was computed using a discrete $480 \times 480 \times$ 240 grid representing a cube of physical dimensions $3700 \times$ $3700 \times 1100 \mathrm{Mm}^{3}$. The equations of mass, energy, and momentum conservation were solved together with the radiative transfer equations adopting realistic equation of state and opacities. The simulation sequence used for the present work consists 


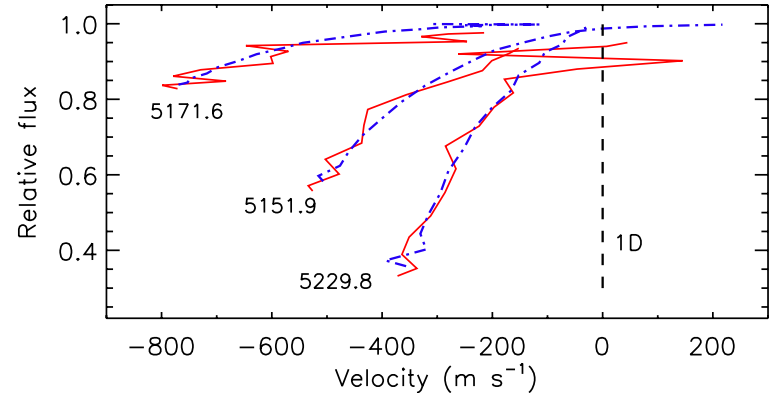

Figure 2. Bisectors of three representative $\mathrm{Fe} I$ lines in the spectrum of HD 122563 (solid lines). Error bars ( $\simeq 30-150 \mathrm{~m} \mathrm{~s}^{-1}$ from core to continuum) have been omitted for clarity. The dot-dashed lines correspond to 3D model predictions. The observed lines in this figure have been shifted to match the theoretical line cores and thus allow a better comparison of bisector shapes. The dashed line at zero velocity represents 1D model predictions.

(A color version of this figure is available in the online journal.)

of 51 snapshots that cover about $150 \mathrm{hr}$ of stellar time and it typically contains about 10 granules at the surface. The evolution timescale of the granules is about $90 \mathrm{hr}$. For more details, see Collet et al. (2009).

We used the 3D model to synthesize all $58 \mathrm{Fe}$ I lines shown in Figure 1. For the line synthesis we adopted a coarser grid of $50 \times 50 \times 125$ points to which we interpolated the original simulation. A number of tests were performed with a higherresolution grid of $100 \times 100 \times 125$ points to ensure that this had no significant impact on the shapes of the synthetic line profiles.

\section{LINE PROFILES: SYNTHETIC VERSUS OBSERVED}

The synthetic 3D profiles were convolved with a Gaussian instrumental profile of FWHM $=1.5 \mathrm{~km} \mathrm{~s}^{-1}$, which corresponds to the spectral resolution of our data. From a $\chi^{2}$ line profile fitting of about $30 \mathrm{Fe}$ I lines with the highest local $\mathrm{S} / \mathrm{N}$, with $V \sin i$ and iron abundance as free parameters, we obtained $V \sin i=3.2 \pm 0.6 \mathrm{~km} \mathrm{~s}^{-1}$. For the $\chi^{2}$ fitting we convolved the disk-integrated profiles (e.g., Gray 1992, p. 370). To calculate the disk-integrated profiles for comparison with the observational data we applied a $V \sin i=3.2 \mathrm{~km} \mathrm{~s}^{-1}$ to the emergent intensities. A detailed abundance analysis of our HD 122563 spectrum using the 3D model will be provided in a later publication. $^{7}$

In Figure 2, we show a comparison of observations and model for three representative $\mathrm{Fe}$ I lines. The observed lines have been shifted by about $+30,+30$, and $-100 \mathrm{~m} \mathrm{~s}^{-1}$ (from strongest to weakest line) to make their core velocities agree with the theoretical values. This allows a better comparison of line bisector shapes because it removes the uncertainties in the measured core wavelengths and rest laboratory wavelengths. We discuss the core wavelength shifts below and show that the artificial shifts applied here are within the observational errors. ${ }^{8}$ The average difference in bisector velocities (model minus observations for all $58 \mathrm{Fe}$ I lines) is $10 \pm 71 \mathrm{~m} \mathrm{~s}^{-1}$

\footnotetext{
7 According to the preliminary analysis by Collet et al. (2009), the 3D-LTE iron abundances determined from $\mathrm{Fe} I$ lines show a trend with excitation potential, going from about $\log \epsilon(\mathrm{Fe})=4.0$ at $\chi=0 \mathrm{eV}$ to $\log \epsilon(\mathrm{Fe})=4.6$ at $\chi=4 \mathrm{eV}$, with some line-to-line scatter comparable to the one from classical $1 \mathrm{D}$ analyses.

8 We avoid the comparison of line bisectors averaged in groups of line strength because this leads to a certain loss of information. The detailed shapes of lines of similar strength can be different due to differences in the properties of the atomic transition involved. Averaging is justified in cases where the $\mathrm{S} / \mathrm{N}$ is low, the spectral lines are severely blended, or the granulation signatures are too weak (e.g., Ramírez et al. 2008).
}
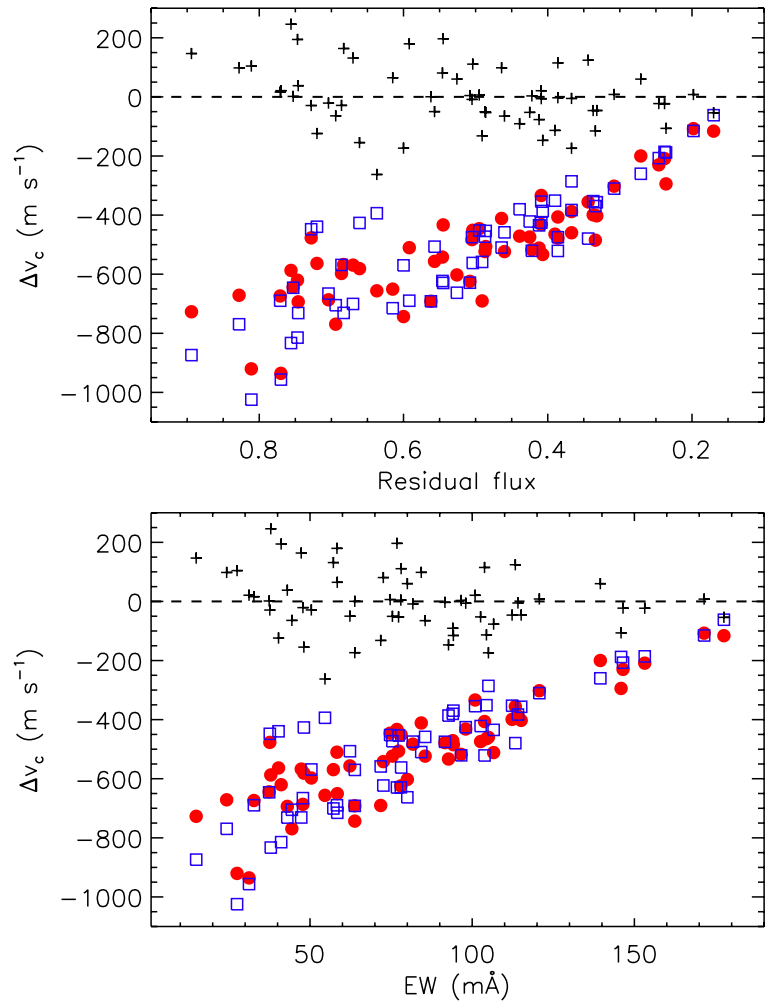

Figure 3. Core wavelength shifts of $58 \mathrm{Fe}$ I lines in the spectrum of HD 122563 (filled circles) and those predicted by the 3D model (open squares) as a function of residual flux (top panel) and equivalent width (bottom panel). The residuals (observation minus model) are shown with crosses. The dashed line is at zero velocity.

(A color version of this figure is available in the online journal.)

(hereafter, error bars correspond to the $1 \sigma$ scatter). This number, however, depends on the relative flux. Near the continuum, the bisector point-by-point difference is about $-30 \pm 145 \mathrm{~m} \mathrm{~s}^{-1}$ while it is $9 \pm 33 \mathrm{~m} \mathrm{~s}^{-1}$ at a residual flux of about 0.2 . At all flux values the difference is consistent with zero within the $1 \sigma$ scatter. The formal error in the observed bisector velocities shows a residual flux dependency remarkably similar to that described above, i.e., it ranges from about 30 to $150 \mathrm{~m} \mathrm{~s}^{-1}$ from core to continuum. Thus, the scatter seen in the differences between theoretical and observed bisectors is fully compatible with the $1 \sigma$ observational errors.

Measurements of core wavelength shifts $\left(\Delta v_{c}\right)$ are in principle more precise than line bisectors, since only a small portion of the line profile is examined, thus lowering the probability of being affected by blends. In Figure 3 we show the relation between $\Delta v_{c}$ and line strength. The so-called third signature of stellar granulation (Gray 2009), i.e., the increased blueshift with decreasing line strength, is clearly detected in the data and very closely reproduced by the 3D model. The third signature is due to the fact that weaker lines form in deeper layers, where the granulation velocities and intensity contrast are larger. The lineto-line scatter seen in Figure 3 increases toward weaker lines, an effect that is present also in the 3D model predictions. It is tempting to conclude that this increased scatter is real, but given the timescale of the evolution of granules in comparison with the duration of the simulation and the possibility that line cores of weaker lines are more difficult to measure (but see below), we must refrain from making a strong statement about this finding. Note also that the variation of the continuous opacity over the wavelength range covered by our data is substantial, which 
introduces wavelength dependent shifts that might contribute to the observed scatter (e.g., Dravins et al. 1981). The difference between observations and model predictions for $\Delta v_{c}$ has a $1 \sigma$ scatter of $104 \mathrm{~m} \mathrm{~s}^{-1}$.

To estimate the error in the measured $\Delta v_{c}$ values we artificially introduced Gaussian noise to the theoretical profiles to simulate the local $\mathrm{S} / \mathrm{N}$ of the data. We took into account the variation of the $\mathrm{S} / \mathrm{N}$ across the line profile since it varies according to the amount of flux detected at each wavelength and it is therefore lower at the line core compared to the local continuum. This experiment was performed after degrading the theoretical profiles to the spectral resolution of our data and convolving it with a rotational profile with the $V \sin i$ we derived for HD 122563. Finally, for each line tested we added in quadrature the error introduced by the uncertainty in the measured laboratory wavelengths $\left(20 \mathrm{~m} \mathrm{~s}^{-1}\right)$ and the estimated error of the wavelength calibration of the data $\left(30 \mathrm{~m} \mathrm{~s}^{-1}\right)$. The $\Delta v_{c}$ values obtained from 1,000 of these tests, averaged over the $58 \mathrm{Fe}$ I lines, have a $1 \sigma$ scatter of $\simeq 108 \mathrm{~m} \mathrm{~s}^{-1}$, nearly independent of line strength but mildly dependent on wavelength, reflecting the variation of the $\mathrm{S} / \mathrm{N}$ across our observed spectrum. This estimated observational error is very similar to the scatter seen in the comparison of model predictions and observations.

\section{SUMMARY AND DISCUSSION}

We have observed the very metal-poor red giant star HD 122563, a primer for studies of the Galactic stellar halo, at very high resolution and high $\mathrm{S} / \mathrm{N}$. The high quality of our data allowed us to measure the asymmetries and core wavelength shifts of a number of $\mathrm{Fe} I$ lines, which clearly revealed the signatures of granulation. A state-of-the-art 3D-LTE radiativehydrodynamic simulation was used to synthesize the same set of lines. Using line profile fitting we derived a projected rotational velocity $V \sin i=3.2 \pm 0.6 \mathrm{~km} \mathrm{~s}^{-1}$ while the analysis of core wavelength shifts allowed us to infer an absolute radial velocity $V_{r}=-25.39 \pm 0.09 \mathrm{~km} \mathrm{~s}^{-1}$. The line bisectors and core wavelength shifts computed from the $3 \mathrm{D}$ model predictions agree with the observations of HD 122563 remarkably well. The average difference between observations and model is consistent with zero within the estimated $1 \sigma$ observational errors. This suggests that the 3D model contains realistic information regarding the atmospheric structure and velocity fields of HD 122563.

Abundance analyses of HD 122563 using our 3D model show that standard 1D estimates of elemental abundances can be off by up to one order of magnitude (Collet et al. 2007, 2009). Qualitatively similar results have been obtained with a simulation computed by an independent group (Ivanauskas et al. 2010; Dobrovolskas et al. 2010), although their abundance corrections tend to be smaller. The large 3D corrections will have an impact on our interpretations of stellar abundance data for halo stars and may lead us to revise their connection with early enrichment of the Galaxy, supernovae yields, and internal mixing processes. The observations presented in this Letter and their comparison to the 3D model predictions suggest that such large 3D effects are reliable and must therefore be seriously considered in all studies of metal-poor stellar abundances.

Admittedly, the largest 3D corrections apply to abundances derived from molecular features whereas we have analyzed only $\mathrm{Fe}_{\mathrm{I}}$ lines. The Fe I lines provide one sampling of the atmospheric structure with a particular weighting over the different structures and velocity fields. Other species, particularly the molecules, will provide different weighting and should in principle be investigated before claiming that the $3 \mathrm{D}$ models are adequately justified for all abundance analyses. Unfortunately, in our spectral range the molecular lines are too weak for this type of work. We note, however, that our Fe I lines form over a wide range of depths, spanning a large portion of the photosphere, including the regions of formation of molecular lines typically used in abundance work $\left(\log \tau_{\text {Ross }} \simeq-2\right)$.

Both our 3D model and line synthesis assumed local thermodynamic equilibrium (LTE), yet the impact of departures from the LTE approximation is likely to be very important at low metallicities (e.g., Asplund 2005). To first-order, however, nonLTE affects the abundances but not the shapes of line profiles. A fully consistent 3D-non-LTE model atmosphere and line formation scheme is currently beyond our reach. Nevertheless, our work represents one step forward in the direction of major improvements to elemental abundance determination techniques.

This work was supported in part by the Robert A. Welch Foundation of Houston, Texas (grant number F-634). We thank the anonymous referee for helping us improve the original manuscript.

\section{REFERENCES}

Allende Prieto, C., Asplund, M., García López, R. J., \& Lambert, D. L. 2002, ApJ, 567, 544

Asplund, M. 2005, ARA\&A, 43, 481

Asplund, M., Nordlund, Å., Trampedach, R., Allende Prieto, C., \& Stein, R. F. 2000, A\&A, 359, 729

Barbuy, B., et al. 2003, ApJ, 588, 1072

Barklem, P. S., Piskunov, N., \& O’Mara, B. J. 2000, A\&AS, 142, 467

Barklem, P. S., Stempels, H. C., Allende Prieto, C., Kochukhov, O. P., Piskunov, N., \& O’Mara, B. J. 2002, A\&A, 385, 951

Cayrel, R., et al. 2004, A\&A, 416, 1117

Collet, R., Asplund, M., \& Trampedach, R. 2006, ApJ, 644, L121

Collet, R., Asplund, M., \& Trampedach, R. 2007, A\&A, 469, 687

Collet, R., Nordlund, A., Asplund, M., Hayek, W., \& Trampedach, R. 2009, Mem. Soc. Astron. Ital., 80, 719

Dobrovolskas, V., Kučinskas, A., Ludwig, H., Caffau, E., Klevas, J., \& Prakapavičius, D. 2010, in 11th Symposium on Nuclei in the Cosmos, NIC XI (arXiv:1010.2507)

Dravins, D. 1990, A\&A, 228, 218

Dravins, D. 2008, A\&A, 492, 199

Dravins, D., Gullberg, D., Lindegren, L., \& Madsen, S. 1999, in ASP Conf. Ser 185, IAU Colloq. 170: Precise Stellar Radial Velocities, ed. J. B. Hearnshaw \& C. D. Scarfe (San Francisco, CA: ASP), 41

Dravins, D., Lindegren, L., \& Nordlund, Å. 1981, A\&A, 96, 345

González Hernández, J. I., Bonifacio, P., Ludwig, H., Caffau, E., Behara, N. T., \& Freytag, B. 2010, A\&A, 519, A46

Gray, D. F. 1983, PASP, 95, 252

Gray, D. F. 1992, The Observation and Analysis of Stellar Photospheres (2nd ed., New York; Cambridge: Cambridge Univ. Press)

Gray, D. F. 2005, PASP, 117, 711

Gray, D. F. 2009, ApJ, 697, 1032

Gray, D. F., Carney, B. W., \& Yong, D. 2008, AJ, 135, 2033

Ivanauskas, A., Kucinskas, A., Ludwig, H., \& Caffau, E. 2010, in 11th Symposium on Nuclei in the Cosmos, NIC XI (arXiv:1010.1722)

Lambert, D. L., Heath, J. E., Lemke, M., \& Drake, J. 1996, ApJS, 103, 183

Lambert, D. L., Sneden, C., \& Ries, L. M. 1974, ApJ, 188, 97

Ludwig, H., Caffau, E., Steffen, M., Freytag, B., Bonifacio, P., \& Kučinskas, A. 2009, Mem. Soc. Astron. Ital., 80, 711

Nave, G., Johansson, S., Learner, R. C. M., Thorne, A. P., \& Brault, J. W. 1994, ApJS, 94, 221

Nesis, A., Hanslmeier, A., Hammer, R., Komm, R., Mattig, W., \& Staiger, J. 1992, A\&A, 253, 561

Nordlund, Å. 1982, A\&A, 107, 1

Nordlund, Å., \& Galsgaard, K. 1995, A 3D MHD Code for Parallel Computers (http://www.astro.ku.dk/ kg/pb/kg/diskg/html/papers/MHD_code.ps.gz)

Nordlund, Å., Stein, R. F., \& Asplund, M. 2009, Living Rev. Sol. Phys., 6, 2 
Ramírez, I., Allende Prieto, C., Koesterke, L., Lambert, D. L., \& Asplund, M. 2009, A\&A, 501, 1087

Ramírez, I., Allende Prieto, C., \& Lambert, D. L. 2008, A\&A, 492, 841

Ramírez, I., \& Meléndez, J. 2005, ApJ, 626, 446

Rutten, R. J., Hammerschlag, R. H., Bettonvil, F. C. M., Sütterlin, P., \& de Wijn, A. G. 2004, A\&A, 413, 1183

Ryan, S. G., Norris, J. E., \& Beers, T. C. 1996, ApJ, 471, 254

Sneden, C. 1973, ApJ, 184, 839
Soubiran, C., Le Campion, J., Cayrel de Strobel, G., \& Caillo, A. 2010, A\&A, 515,111

Steffen, M., \& Freytag, B. 1991, in Reviews in Modern Astronomy, Vol. 4, ed. G. Klare (New York: Spring-Verlag), 43

Tull, R. G., MacQueen, P. J., Sneden, C., \& Lambert, D. L. 1995, PASP, 107 251

van Leeuwen, F. 2007, A\&A, 474, 653

Wallerstein, G., Greenstein, J. L., Parker, R., Helfer, H. L., \& Aller, L. H. 1963, ApJ, 137, 280 